\title{
Dynamic Tissue Perfusion Measurement: A New Tool for Characterizing Renal Perfusion in Renal Cell Carcinoma Patients
}

\author{
Clemens Rosenbaum ${ }^{\mathrm{a}}$ Sven Wach ${ }^{\mathrm{a}}$ Frank Kunath $^{\mathrm{a}}$ Bernd Wullich $^{\mathrm{a}}$ \\ Thomas Scholbach $^{b}$ Dirk G. Engehausen ${ }^{a}$ \\ ${ }^{a}$ Department of Urology, Universitätsklinikum Erlangen, Friedrich-Alexander Universität Erlangen-Nürnberg, \\ Erlangen, and ${ }^{b}$ Clinic for Pediatrics and Adolescent Medicine, Klinikum Chemnitz, Chemnitz, Germany
}

\section{Key Words}

Kidney $\cdot$ Renal cell carcinoma Dynamic tissue perfusion measurement $\cdot$ Color Doppler ultrasound

\begin{abstract}
Introduction: Renal cell carcinoma (RCC) is characterized by intense angiogenesis with hyperexpression of proangiogenic factors. This study explored the potential of dynamic tissue perfusion measurement (DTPM) to detect differences in tissue perfusion between kidneys with RCC and corresponding healthy kidneys. Patients and Methods: 30 patients with unilateral, histologically confirmed RCC underwent DTPM by color Doppler ultrasound. Before scheduled surgery, Doppler ultrasound data were acquired from four transverse areas of the affected kidney and the contralateral healthy kidney. Doppler ultrasound data were recorded over a 10-second period and characteristic tissue perfusion parameters were determined. Results: The kidneys with RCC displayed characteristic changes in perfusion parameters. A significant increase in signal intensity and a significant decrease in flow resistance were noted. A combination of several DTPM parameters was used to distinguish correctly between kidneys bearing RCC or healthy kidneys with up to $75 \%$ accuracy. There was no association between the perfusion parameters and the pathological characteristics of the respective tu-
\end{abstract}

mors. Conclusions: DTPM is a promising tool for the evaluation of whole-organ tissue perfusion. This study demonstrates the feasibility of performing DTPM measurements in kidneys bearing RCC lesions. In tumors that are characterized by extensive neovascularization, this method has the potential to be a valuable diagnostic tool.

Copyright $\odot 2012$ S. Karger AG, Basel

\section{Introduction}

Approximately 3\% of all diagnosed cancers are renal cell carcinomas (RCCs). Of the urinary cancers, RCC is the third most common malignancy [1]. The incidence of RCC has risen over the recent years, probably because of the increased usage of abdominal imaging [2]. Of the incidentally found solid lesions of the kidney, up to $20 \%$ of the cases are benign [3]. Diagnostic procedures, such as abdominal ultrasound (US), computed tomography and magnetic resonance imaging, are commonly utilized. Of these methods, US is a time-efficient and non-invasive imaging technique. Furthermore, the necessary equipment is commonly available. Efforts have been under-

C. Rosenbaum and S. Wach contributed equally.

\section{KARGER}

Fax +4161306 1234

E-Mail karger@karger.ch

www.karger.com (c) 2012 S. Karger AG, Basel

0042-1138/13/0901-0087\$38.00/0

Accessible online at:

www.karger.com/uin
Dirk G. Engehausen

Department of Urology, University Hospital Erlangen

Krankenhausstrasse 12

DE-91054 Erlangen (Germany)

E-Mail dirk.engehausen@uk-erlangen.de 
taken to improve the diagnostic potential of US for differentiating between benign and malign lesions or characterizing vascularity [4-6]. RCC is characterized by a high degree of vascularization and pathologically increased blood flow $[5,7]$. Hereby, the overexpression of proangiogenic factors and intense angiogenesis are a hallmark of many RCCs [8-10]. Recently, antiangiogenic drugs, such as tyrosine kinase inhibitors, have been approved for first-line therapy of RCC $[11,12]$. Because of these reasons, US assessment of blood flow may hold a significant potential not only for diagnostic purposes but also for monitoring therapy. The conventional color Doppler US has limitations in resolving intratumor vascularization [13]. Although contrast-enhanced US is an invasive procedure, it has already been successfully applied for measuring tissue perfusion in primary tumor lesions [14] or for monitoring therapy response [15].

In this study, we used conventional color Doppler US and applied a novel dynamic tissue perfusion measurement (DTPM) technique to assess for the first time wholeorgan perfusion in kidneys bearing RCC. The kidneys with histologically confirmed RCC showed significant changes in several perfusion parameters compared to corresponding healthy kidneys.

\section{Patients and Methods}

\section{Study Population}

Between May 2009 and June 2010, 32 patients with focal renal lesions were included in this study. All patients gave informed consent prior to examination. Renal lesions were confirmed by computed tomography imaging. The patients were hospitalized for partial or radical nephrectomy. Two patients were excluded because of the following reasons: absence of the contralateral kidney $(\mathrm{n}=1)$ or previous kidney transplant $(\mathrm{n}=1)$. In the final evaluation, 30 patients were retrospectively analyzed.

Of these cases, histopathological examination confirmed 25 cases of clear cell RCC (83.4\%), 4 cases of papillary RCC (13.3\%) and 1 case of chromophobe RCC (3.3\%). Detailed information regarding the composition of the study population and the histopathological classification of the RCC tumors is shown in table 1.

\section{Color Doppler Sonography}

The patients were examined by Doppler US the day before scheduled surgery. Investigation of the kidney was performed with a 4- to 8-MHz curved probe using Pro Focus 2202 US equipment (BK Medical, Herlev, Denmark). Four transverse sections per kidney (two through the upper pole and two through the lower pole; fig. 1) were recorded. The presettings of the US equipment remained constant. The frequency was set to $5.5 \mathrm{MHz}$, and the color gain was fixed by the presetting and remained constant. To minimize artifacts caused by organ movement, the patients were asked to arrest breathing during image acquisition. A total of $166 \mathrm{im}$ ages were acquired during the $10 \mathrm{~s}$ of recording time. The images

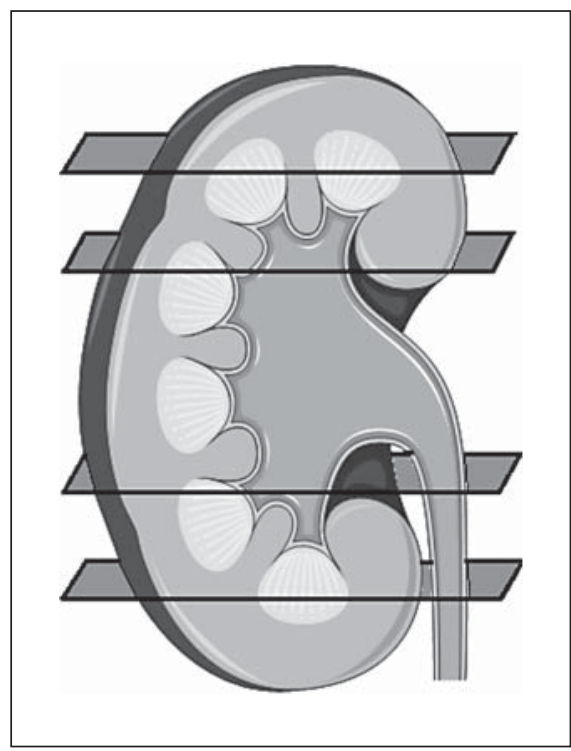

Fig. 1. Schematic of the Doppler US procedure. Doppler US data were obtained from four transverse sections of the kidney. The figure was produced with permission using Servier Medical Art.

Table 1. Patient and tumor characteristics

\begin{tabular}{ll}
\hline Preoperative features & 20 \\
Male & 10 \\
Female & $65.7(41-82)$ \\
Age, median (range) & 16 \\
Right-sided tumor & 14 \\
Left-sided tumor & \\
T stage & 10 \\
pT1a & 8 \\
pT1b & 8 \\
pT3a & 4 \\
pT3b & 4 \\
Fuhrman nuclear grade & 14 \\
Grade 1 & 12 \\
Grade 2 & \\
Grade 3 & 2.1 \\
Tumor size, cm & 6.3 \\
Minimum & 16 \\
Mean & \\
Maximum &
\end{tabular}

were immediately checked for movement artifacts, and the measurements were repeated if necessary. The recording time of $10 \mathrm{~s}$ was chosen to acquire US images during multiple cardiac cycles.

Dynamic Tissue Perfusion Measurement

Digital color Doppler sonographic videos (DICOM format) were analyzed with PixelFlux ${ }^{\circledR}$ software (Chameleon Software, 

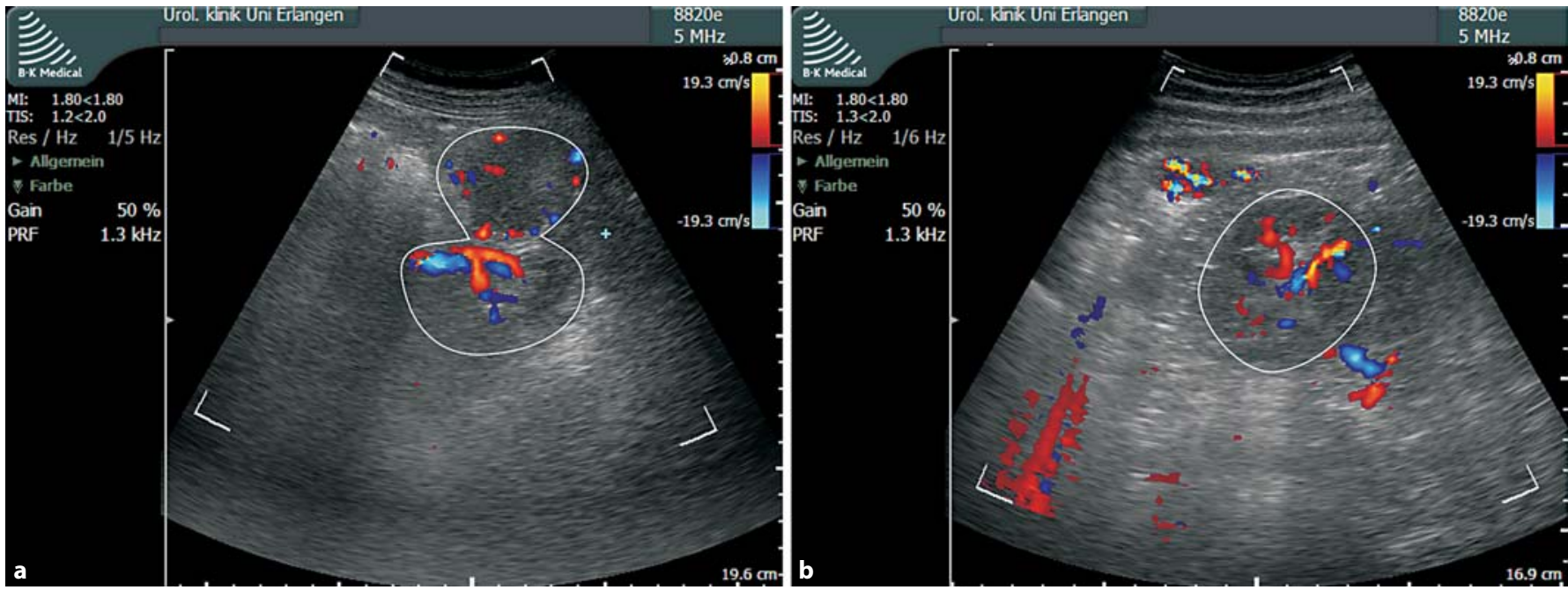

Fig. 2. Color Doppler US-based flow measurement. a Blood flow in a kidney with RCC. b Blood flow in the respective contralateral kidney. The position of the kidney and the RCC lesion, if present, is indicated by white circles.

Leipzig, Germany). For every patient, eight datasets were available: four transverse sections each for the affected kidney and for the healthy contralateral kidney. Briefly, after size calibration with a standardized size marker, a region of interest (ROI) was defined in every dataset, encompassing the entire kidney. RCC lesions were included in the ROI only when they were located within the specified transverse section. The direction and velocity of the dynamic blood flow was visualized by encoding different shades of red (blood flow directed towards the US probe) or blue (blood flow directed away from the US probe) pixels. Representative images of a kidney bearing RCC and the respective contralateral kidney are shown in figure 2. During the examination, the US probe was aligned in such a way that arterial blood flow was predominately directed toward the probe (red) and venous blood flow directed from the probe (blue). The maximum encodable flow velocity was set to $\pm 19.3 \mathrm{~cm} / \mathrm{s}$. Individual cardiac cycles were detected automatically by the PixelFlux ${ }^{\circledR}$ software and all complete cardiac cycles were the basis for further calculations. We increased the recording time from $3 \mathrm{~s} \mathrm{[16-19]} \mathrm{to} 10 \mathrm{~s}$. As this was the first time, this technique was applied to kidneys bearing RCC, a prolonged recording time was chosen in order to receive valid Doppler US data from at least one complete cardiac cycle. By measuring the perfused area and individual flow velocities in the ROI, several characteristic perfusion parameters were determined. The area (A) of displayed vessels denotes the area $\left(\mathrm{cm}^{2}\right)$ that was perfused, as stratified according to blood flow direction. The tissue pulsatility index (TPI) of all colored pixels was calculated as the difference of mean maximum systolic ( $\mathrm{v}$ sys) and mean minimum diastolic ( $v$ dia) blood flow velocity divided by the average blood flow velocity (v average); TPI = (v sys $-\mathrm{v}$ dia $) / \mathrm{v}$ average, where velocity refers to all colored pixels inside the ROI. This parameter could also be stratified according to blood flow direction. The tissue resistance index (TRI) of the entire ROI denotes, similar to the TPI, the difference between the maximum systolic ( $\mathrm{v}$ sys) and the minimum diastolic ( $\mathrm{v}$ dia) blood flow velocity divided by the maximum sys- tolic flow velocity ( $\mathrm{v}$ sys); TPI $=(\mathrm{v}$ sys $-\mathrm{v}$ dia $) / \mathrm{v}$ sys. The signal intensity $(\mathrm{F})$ is a global measure of tissue perfusion. ' $\mathrm{F}$ ' is calculated by multiplying the vessel area $\mathrm{A}$ of all vessels inside the ROI by the mean flow velocity (v) of all colored pixels and division by the area of the ROI. Each perfusion parameter was derived from perfused areas and flow velocities detected during a complete cardiac cycle. If multiple cardiac cycles were detected in the recording of $10 \mathrm{~s}$ per transverse section, the parameters represent the average values based on all complete cardiac cycles. To obtain a measurement for the whole organ, we averaged the calculations of the four transverse sections recorded for the same organ.

\section{Statistical Analyses}

To compare tissue perfusion characteristics between kidneys with RCC lesions and healthy contralateral kidneys, we used nonparametric Wilcoxon-matched pairs test statistics. All tests were performed as two-sided, and p values $\leq 0.05$ were considered to be significant. Statistical analyses were performed using GraphPad Prism 4.0 software (GraphPad, Inc., La Jolla, Calif., USA).

\section{Results}

\section{Tissue Perfusion}

First, we were interested whether the presence of RCC changes the perfusion in the affected organ. The day before scheduled surgery, Doppler US measurements were recorded for the affected organ and the contralateral healthy organ. The calculation of characteristic tissue perfusion parameters was performed retrospectively after the recruitment period. We did not stratify the patient cohort according to histological subtypes because this approach 


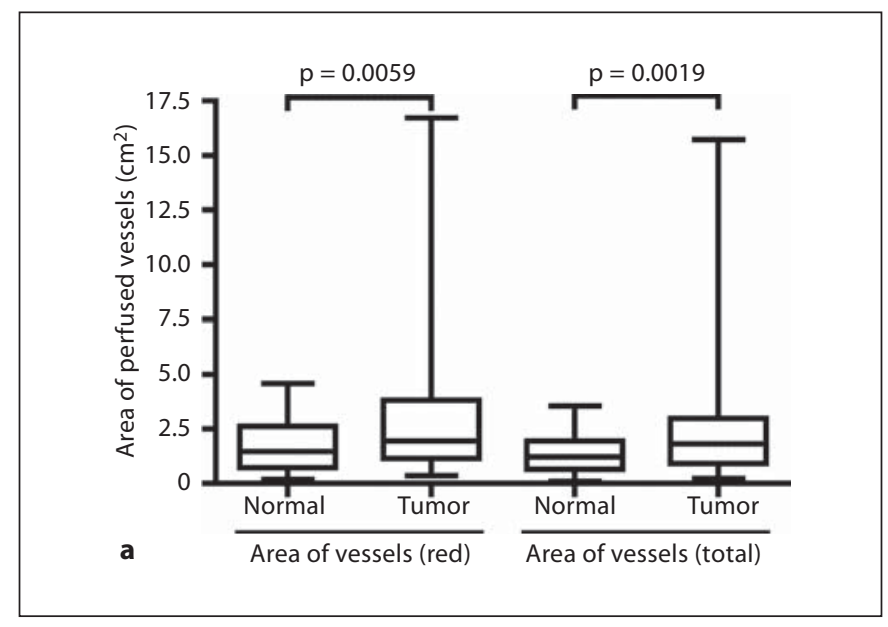

Fig. 3. Renal perfusion parameters. a Area of perfused vessels. b Signal intensity. The data are representative of 30 kidneys with RCC and 30 healthy kidneys. The data are presented as first and third quartile (box) with the median marked as a horizontal bar.

would result in small sample groups. When we examined the arterial blood flow, as determined by the area displaying red pixels, we noticed that the kidneys with RCC showed a higher proportion of arterial blood flow. The area showing arterial blood flow was 1.33 -fold larger in kidneys with RCC (median $1.946 \mathrm{~cm}^{2}$, range 0.354-16.37 in RCC vs. $1.467 \mathrm{~cm}^{2}$, range $0.177-4.589$ in healthy kidneys; $p=0.006$; fig. $3 \mathrm{a})$. A similar result was noted when we examined the total perfused area $\left(A_{\text {total }}\right)$ irrespective of the direction of the blood flow. In this case, affected kidneys displayed a perfused area that was 1.49 -fold larger than in healthy control organs (median $1.821 \mathrm{~cm}^{2}$, range $0.224-15.72$ vs. $1.221 \mathrm{~cm}^{2}$, range $0.100-3.556 ; \mathrm{p}=$ 0.002 ; fig. 3a). This result indicates that the number or size of blood vessels is increased in kidneys with RCC. To receive a more comparable measure of perfusion, we also calculated the global signal intensity, which incorporates not only the perfused area and flow velocity but is also independent of the ROI. The maximum signal intensity $\left(\mathrm{I}_{\max }\right)$, as determined during systolic blood flow, was elevated up to 1.67 -fold in affected kidneys (median 8.006 $\mathrm{cm} / \mathrm{s}$, range $2.624-15.09$ vs. $4.787 \mathrm{~cm} / \mathrm{s}$, range 1.162-9,796; $\mathrm{p}<0.0001$; fig. $3 \mathrm{~b}$ ), and the average signal intensity during a complete cardiac cycle was likewise elevated up to 1.37 fold (median $0.838 \mathrm{~cm} / \mathrm{s}$, range $0.257-2.989$ vs. $0.611 \mathrm{~cm} / \mathrm{s}$, range $0.114-1.66 ; p=0.004$; fig. $3 b$ ). These results demonstrate that RCC shows not only an increase in blood vessel density but also an increase in global perfusion.

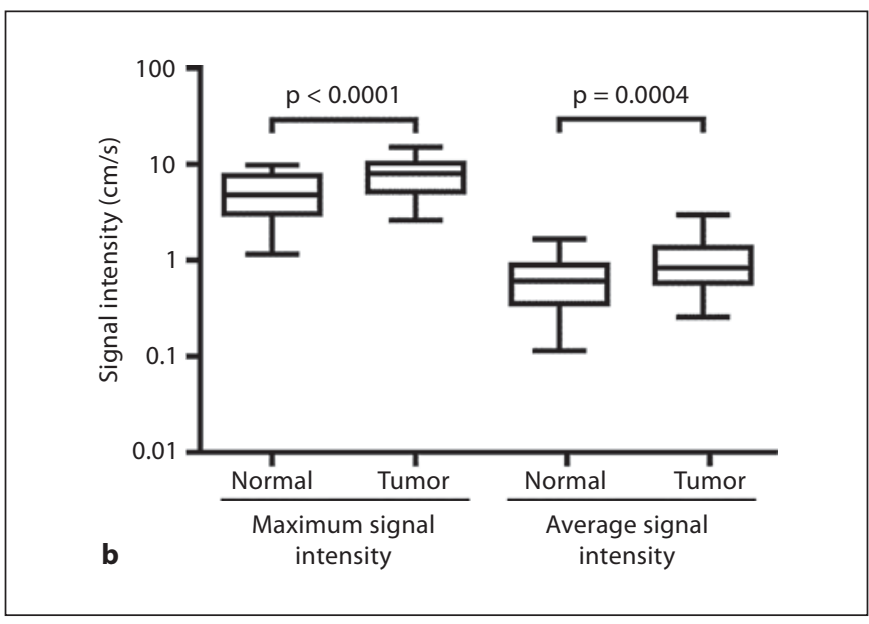

The whiskers represent the maximum and minimum values of the dataset. $\mathrm{p}$ values are derived from Wilcoxon-matched pairs test statistics.
As papillary RCC is the RCC entity most commonly associated with hypovascular features [20], we also compared characteristic tissue perfusion parameters between the subgroups of clear cell and papillary RCC. In the areas displaying blood flow, both arterial and total areas are reduced in papillary RCC (table 2). These results demonstrated that hypovascular features commonly associated with pRCC might also be associated with decreased renal perfusion in pRCC compared to clear cell RCC.

\section{Peripheral Flow Resistance}

TRI and TPI are more sophisticated measures of peripheral flow resistance than the common RI or PI. These values are considered to be associated with physiological changes, such as vascular spread, vascular branching or interstitial pressure [18]. When comparing these measures, we detected several changes between kidneys bearing RCC and healthy control kidneys. Both TRI and TPI for blue (predominantly venous) vessels are significantly reduced in organs with RCC. Specifically, TRI $=0.803$, range $0.52-1.001$ in normal kidneys vs. 0.702, range $0.418-1.0$ in RCC; $\mathrm{p}=0.003$, and TPI $=1.662$, range $0.686-3.754$ in normal kidneys vs. 1.281, range $0.512-$ 2.946 in RCC; $p=0.006$ (fig. 4). A similar result was obtained when examining the red (predominantly arterial) vessels. TRI was likewise significantly reduced (TRI = 0.662 , range $0.502-1.0$ in normal kidneys vs. 0.624 , range $0.351-0.826$ in RCC, $p=0.043)$. The TPI of red vessels was not significantly reduced, and we noticed a reduction of 


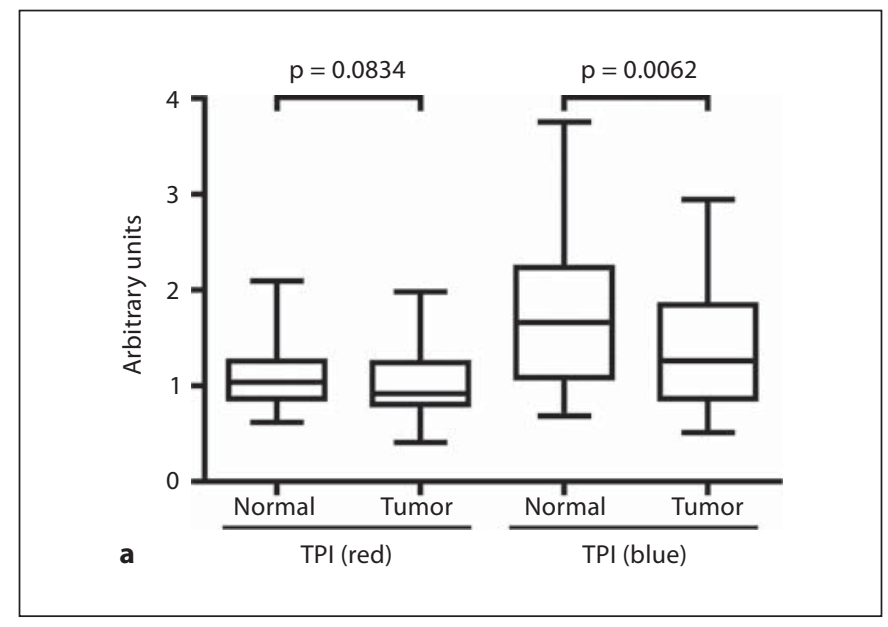

Fig. 4. Renal perfusion parameters. a TPI stratified according to arterial (red) and venous (blue) blood flow. b TRI stratified according to arterial (red) and venous (blue) blood flow. The data are representative for 30 kidneys with RCC and 30 healthy kid-

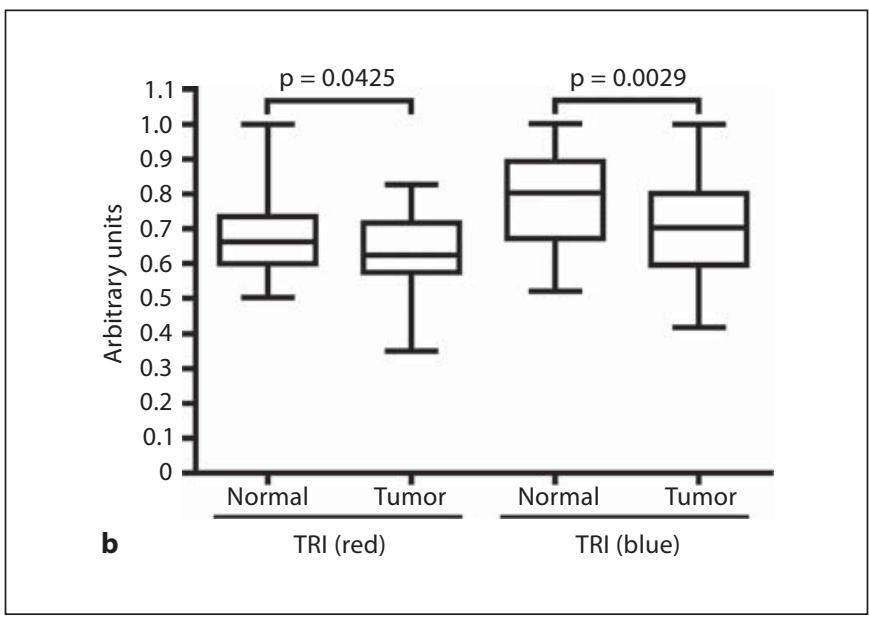

neys. The data are presented as first and third quartile (box) with the median marked as a horizontal bar. The whiskers represent the maximum and minimum values of the dataset. $p$ values are derived from Wilcoxon-matched pairs test statistics.

Table 2. Statistical analyses: tissue perfusion parameters in normal and RCC kidneys as well as in ccRCC and pRCC subgroups

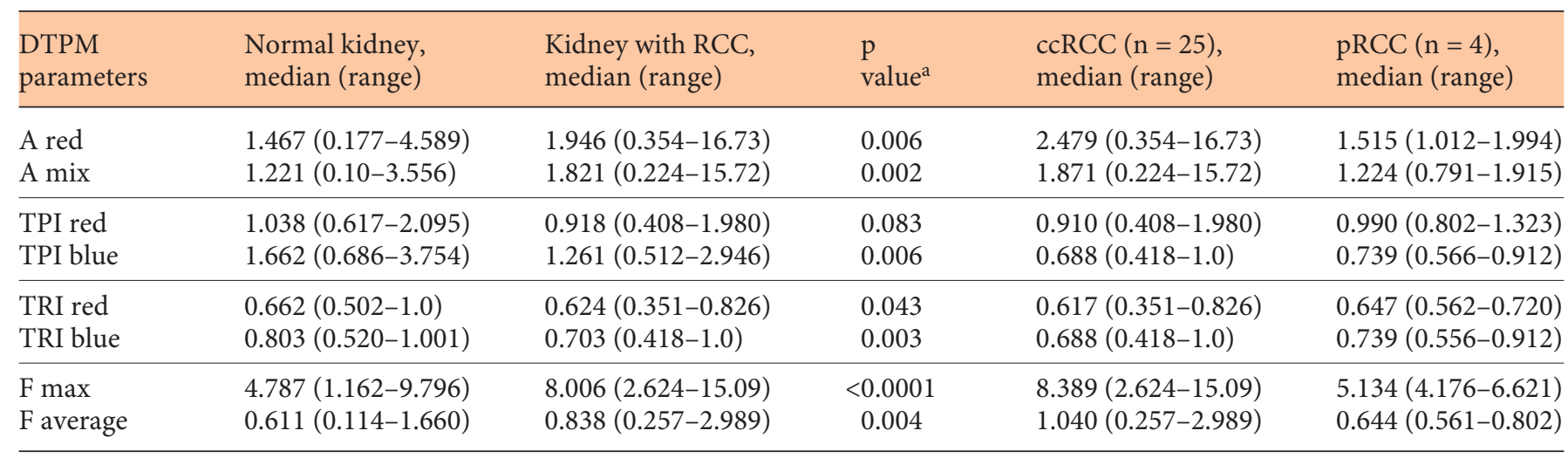

${ }^{a} \mathrm{p}$ values were derived by Wilcoxon-matched pairs test statistics.

only $2 \%$ for this parameter. Altogether, these results indicate that RCC leads to a reduction in peripheral flow resistance in kidneys bearing RCC.

Again, we compared these flow resistance parameters in the subgroups of clear cell and papillary RCC (table 2). Here, the flow resistance parameters did not differ to a great extent between the histologic subtypes of RCC.

\section{Diagnostic Capabilities of Perfusion Parameters}

As several perfusion parameters were altered in kidneys with RCC, we examined whether these parameters are capable of distinguishing between kidneys with or without RCC. All available parameters were tested as potential markers in a binary logistic regression analysis to determine their diagnostic properties. The best single discriminating factor was the $\mathrm{I}_{\max }$ with $66.7 \%$ of cases classified correctly. To select the optimal combination of perfusion parameters with the highest predictive potential, we used a binary logistic regression analysis and applied a backward elimination algorithm. A combination of the parameters $I_{\max }$ and $A_{\text {total }}$ was identified and was able to correctly predict the presence or absence of RCC in $75 \%$ of all organs. 


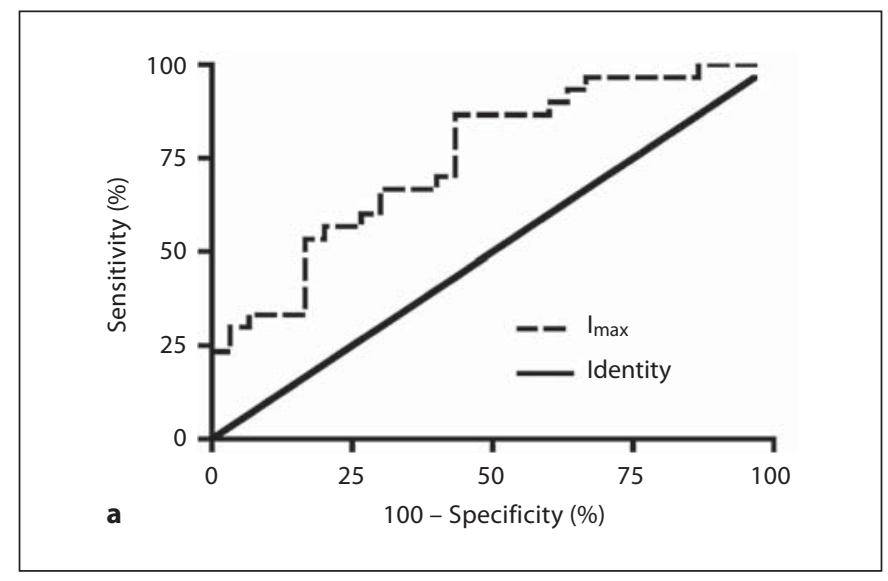

Fig. 5. ROC curve of sensitivity and specificity of RCC detection. The two best single discriminating factors, $\mathrm{I}_{\max }(\mathbf{a})$ and $\mathrm{A}_{\text {total }}(\mathbf{b})$, and the combination of both factors (c) are shown.

To characterize further the capability of the selected parameters to distinguish between tumor and non-tumor samples, we performed a receiver operator characteristics (ROC) analysis. When analyzing $I_{\max }$, the resulting ROC curve revealed an area under the curve (AUC) of 0.75 ( $p<0.01$; fig. $5 \mathrm{a}$ ). In the case of the $A_{\text {total }}$, the AUC was 0.66 ( $p=0.03$; fig. $5 b$ ). Combining both parameters resulted in an AUC of $0.78(\mathrm{p}<0.01$; fig. $5 \mathrm{c})$. These results demonstrate that the perfusion parameters can distinguish between affected and normal kidneys.

\section{Associations with Clinicopathological Features}

We next analyzed whether any perfusion parameter correlated with clinicopathological parameters. We used a non-parametric bivariate correlation to test for an association with the clinicopathological parameters of $\mathrm{pT}$ stage, Fuhrman grade and tumor size (table 1). In no case did we find any association of tissue perfusion parameters of the affected organ and clinicopathological features of the tumor (data not shown). A similar result was ob-
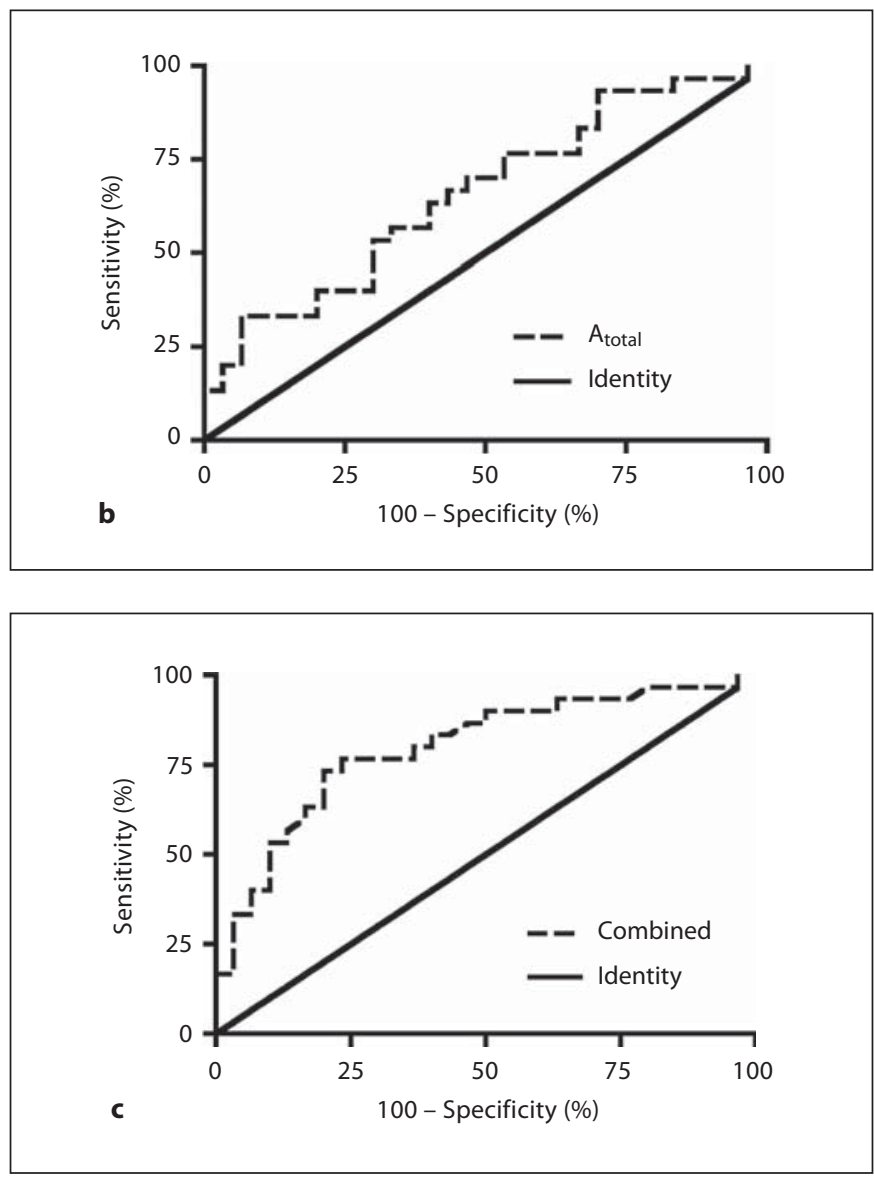

tained when we analyzed the subgroups of clear cell RCC $(\mathrm{n}=25)$ and papillary RCC $(\mathrm{n}=4)$. We could not detect any association of tissue perfusion parameters and the defined clinicopathological characteristics in either the subgroup of clear cell RCC or in the subgroup of papillary RCC (data not shown). These results point toward the conclusion that whole-organ perfusion parameters are independent predictors of the presence of an RCC lesion and that they are not affected by tumor stage or size.

\section{Discussion}

We recorded kidney perfusion by color Doppler US and performed DTPMs. The main advantage of DTPM is its ability to dynamically measure blood flow parameters for several cardiac cycles. This feature is unique among other US-based imaging techniques. As contrast agents are unnecessary for this type of examination, which is cost-efficient and non-invasive, the observation time is not restrict- 
ed and the examination can be repeated as often as needed. To gain consistent and robust data, we increased the recording time from $3 \mathrm{~s}$ [16-19] to $10 \mathrm{~s}$. Thus, the data presented here are average perfusion parameters from several cardiac cycles. Using this information, we were able to calculate various parameters, such as vessel area and dynamic flow velocities, to judge the overall perfusion status of a kidney.

We noticed several changes in renal perfusion parameters. Hereby, signal intensity (the maximum intensity during the observation period or the average intensity during several cardiac cycles) was considered to be the most objective measure because this parameter incorporates the area of perfused vessels and flow velocities. It has already been described as a general measure of tissue perfusion [18]. Tumor-bearing kidneys displayed a significantly increased signal intensity. As differences in signal intensities between affected and unaffected kidneys are more pronounced than the vessel area alone, we concluded that both increased vascularity and increased flow velocities contribute to the differences in signal intensity. The relevance of signal intensity is further demonstrated by the fact that there is a correlation of higher signal intensity with elevated tissue oxygenation in cervical lymph node metastases [18] and with local inflammation in patients with Crohn's disease [17].

Furthermore, in addition to the aforementioned parameters, we were able to define a TPI and a TRI. Similar perfusion parameters have been used before to describe intraorgan vessel resistance [21]. A higher intrarenal vessel resistance has been described for several pathological changes, such as tubulointerstitial and vascular nephropathies [6], edema or focal fibrosis [22]. Moreover, an association of decreased vessel resistance with advanced stages of RCC [23] or with a more severe histological grade [24] has already been reported. The measures of tissue pulsatility and resistance index used in this study do not only measure larger vessels, such as interlobular arteries, but also incorporate information regarding the mechanical properties of the surrounding tissue. In affected kidneys, we noted a decrease in both the tissue pulsatility and resistance index. Both parameters were decreased in an analysis of cervical lymph node metastases from squamous cell head and neck cancer [18]. Although this method is not able to clearly differentiate between preexisting and newly formed vessels, it can be regarded as an indicator of differences in vascular spread, branching, caliber tapering or interstitial pressure [18].

As RCC displays notably few clinical symptoms in its early stages, it is prone to late detection when patients are in locally advanced stages [25]. Multidetector computer tomography as the gold standard of diagnosis has already dramatically improved patient outcome by the detection of small renal masses [26]. US-based imaging techniques have recently been tested for monitoring therapy response in RCC $[15,27]$ but rarely in a diagnostic setting [5]. In our patient cohort, we directly compared DTPM parameters from kidneys with histologically confirmed RCC and contralateral healthy organs. In this study, the best discriminating DTPM parameter could correctly predict the status of the respective organ with an accuracy of $66.7 \%$; a combination of multiple parameters improved the prediction rate to $75 \%$. Prospective screening studies are warranted to determine the diagnostic value of DTPM. In the case of suspected RCC, $10-20 \%$ of renal masses are identified as benign lesions [28-31]. Further experiments are needed to investigate tissue perfusion parameters in kidneys with confirmed benign lesions and to investigate the potential of DTPM to discriminate between benign lesions and RCC.

It is of note that none of the recorded DTPM parameters displayed any association with pathological parameters, such as pT stage, Fuhrman grade or tumor size. These data point toward the assumption that RCC alters renal vascularization to the same extent, irrespective of tumor size or stage.

\section{Conclusions}

DTPM is a useful tool in quantifying various tissue perfusion parameters. In kidneys bearing RCC, we detected several characteristic changes in the recorded parameters that might be indicative of enhanced tissue perfusion in RCC. DTPM measurements are able to discriminate between healthy kidneys and RCC with high accuracy. As DTPM is non-invasive, cost-efficient and easy to perform, this method has the potential to be a valuable tool in the diagnosis of RCC.

\section{Acknowledgment}

We would like to thank American Journal Experts for editorial assistance.

\section{Disclosure Statement}

The authors have no conflicts of interest to disclose. 


\section{References}

1 Hock LM, Lynch J, Balaji KC: Increasing incidence of all stages of kidney cancer in the last two decades in the United States: an analysis of surveillance, epidemiology and end results program data. J Urol 2002;167: 57-60.

2 Chow WH, Devesa SS, Warren JL, Fraumeni JF Jr: Rising incidence of renal cell cancer in the United States. JAMA 1999;281:16281631.

3 Chawla SN, Crispen PL, Hanlon AL, Greenberg RE, Chen DY, Uzzo RG: The natural history of observed enhancing renal masses: meta-analysis and review of the world literature. J Urol 2006;175:425-431.

4 Quaia E, Siracusano S, Bertolotto M, Monduzzi M, Mucelli RP: Characterization of renal tumours with pulse inversion harmonic imaging by intermittent high mechanical index technique: initial results. Eur Radiol 2003;13:1402-1412.

5 Tamai H, Takiguchi Y, Oka M, Shingaki N, Enomoto S, Shiraki T, Furuta M, Inoue I, Iguchi M, Yanaoka K, Arii K, Shimizu Y, Nakata H, Shinka T, Sanke T, Ichinose M: Contrast-enhanced ultrasonography in the diagnosis of solid renal tumors. J Ultrasound Med 2005;24:1635-1640.

6 Angelini P, Mancini A, Cuzzola C, Petrarulo F: Role of the ultrasound and color-Doppler in the progression of the renal damage. G Ital Nefrol 2008;25:441-448.

7 Reese JH: Renal cell carcinoma. Curr Opin Oncol 1992;4:427-434.

8 Nanus DM, Schmitz-Drager BJ, Motzer RJ, Lee AC, Vlamis V, Cordon-Cardo C, Albino AP, Reuter VE: Expression of basic fibroblast growth factor in primary human renal tumors: correlation with poor survival. J Natl Cancer Inst 1993;85:1597-1599.

9 Berger DP, Herbstritt L, Dengler WA, Marme D, Mertelsmann R, Fiebig HH: Vascular endothelial growth factor mRNA expression in human tumor models of different histologies. Ann Oncol 1995;6:817-825.

10 Mulders P: Vascular endothelial growth factor and mTOR pathways in renal cell carcinoma: differences and synergies of two targeted mechanisms. BJU Int 2009; 104:15851589.
11 Bussolati B, Satolli MA, Camussi G: The role of angiogenesis in renal carcinoma. G Ital Nefrol 2008;25:297-305.

12 Vasudev NS, Larkin JM: Tyrosine kinase inhibitors in the treatment of advanced renal cell carcinoma: focus on pazopanib. Clin Med Insights Oncol 2011;5:333-342.

13 Xu ZF, Xu HX, Xie XY, Liu GJ, Zheng YL, Liang JY, Lu MD: Renal cell carcinoma: realtime contrast-enhanced ultrasound findings. Abdom Imaging 2009;35:750-756.

14 Gerst S, Hann LE, Li D, Gonen M, Tickoo S, Sohn MJ, Russo P: Evaluation of renal masses with contrast-enhanced ultrasound: initial experience. AJR Am J Roentgenol 2011; 197:897-906.

15 Lassau N, Koscielny S, Albiges L, Chami L, Benatsou B, Chebil M, Roche A, Escudier BJ: Metastatic renal cell carcinoma treated with sunitinib: early evaluation of treatment response using dynamic contrast-enhanced ultrasonography. Clin Cancer Res 2010;16: 1216-1225.

16 Scholbach T, Dimos I, Scholbach J: A new method of color Doppler perfusion measurement via dynamic sonographic signal quantification in renal parenchyma. Nephron Physiol 2004;96:p99-p104.

17 Scholbach T, Herrero I, Scholbach J: Dynamic color Doppler sonography of intestinal wall in patients with Crohn disease compared with healthy subjects. J Pediatr Gastroenterol Nutr 2004;39:524-528.

18 Scholbach T, Scholbach J, Krombach GA, Gagel B, Maneschi P, Di Martino E: New method of dynamic color Doppler signal quantification in metastatic lymph nodes compared to direct polarographic measurements of tissue oxygenation. Int J Cancer 2005;114:957-962.

19 Scholbach T, Girelli E, Scholbach J: Tissue pulsatility index: a new parameter to evaluate renal transplant perfusion. Transplantation 2006;81:751-755.

20 Onishi T, Oishi Y, Goto H, Yanada S, Abe K: Histological features of hypovascular or avascular renal cell carcinoma: the experience at four university hospitals. Int J Clin Oncol 2002;7:159-164.

21 Soliman NA, Saif A, Hamid AA, Moustafa $H$ : Renal duplex Doppler ultrasonography in patients with recurrent urinary tract infection. Saudi J Kidney Dis Transpl 2009;20: 816-821.
22 Mostbeck GH, Kain R, Mallek R, Derfler K, Walter R, Havelec L, Tscholakoff D: Duplex Doppler sonography in renal parenchymal disease. Histopathologic correlation. J Ultrasound Med 1991;10:189-194.

23 Alcazar JL, Galan MJ, Jurado M, Lopez-Garcia G: Intratumoral blood flow analysis in endometrial carcinoma: correlation with tumor characteristics and risk for recurrence. Gynecol Oncol 2002;84:258-262.

24 Greco P, Vimercati A, Cormio G, Loverro G, Selvaggi L: Intratumoral blood flow characteristics and prognostic factors in patients with endometrial carcinoma. Arch Gynecol Obstet 2002;267:1-3.

25 Ganti S, Weiss RH: Urine metabolomics for kidney cancer detection and biomarker discovery. Urol Oncol 2011;29:551-557.

26 Johnson PT, Horton KM, Fishman EK: How not to miss or mischaracterize a renal cell carcinoma: protocols, pearls, and pitfalls. AJR Am J Roentgenol 2010;194:W307-W315.

27 Lassau N, Chebil M, Chami L, Bidault S, Girard E, Roche A: Dynamic contrast-enhanced ultrasonography: a new tool for the early evaluation of antiangiogenic treatment. Target Oncol 2010;5:53-58.

28 Duchene DA, Lotan Y, Cadeddu JA, Sagalowsky AI, Koeneman KS: Histopathology of surgically managed renal tumors: analysis of a contemporary series. Urology 2003;62: 827-830.

29 Snyder ME, Bach A, Kattan MW, Raj GV, Reuter VE, Russo P: Incidence of benign lesions for clinically localized renal masses smaller than $7 \mathrm{~cm}$ in radiological diameter: influence of sex. J Urol 2006;176:2391-2396.

30 Fujii Y, Komai Y, Saito K, Iimura Y, Yonese J, Kawakami S, Ishikawa Y, Kumagai J, Kihara K, Fukui I: Incidence of benign pathologic lesions at partial nephrectomy for presumed RCC renal masses: Japanese dual-center experience with 176 consecutive patients. Urology 2008;72:598-602.

31 Xiong YH, Zhang ZL, Li YH, Liu ZW, Hou GL, Liu Q, Yun JP, Zhang XQ, Zhou FJ: Benign pathological findings in 303 Chinese patients undergoing surgery for presumed localized renal cell carcinoma. Int J Urol 2010;17:517-521. 